\title{
A Case of Churg-Strauss Syndrome
}

\author{
Ji Hoon Choi, M.D., In Su Ahn, M.D., Hee Bong Lee, M.D., Chun Wook Park, M.D., \\ Cheol Heon Lee, M.D., Hye Kunng Ahn, M.D. ${ }^{1}$
}

Departments of Dermatology and ${ }^{1}$ Pathology, College of Medicine, Hallym University, Seoul, Korea

Churg-Strauss syndrome (CSS) or allergic granulomatosis angiitis is a rare primary vasculitic disease. CSS can be diagnosed by the presence of any four or more of the six criteria, which include asthma, eosinophilia greater than $10 \%$, paranasal sinusitis, pulmonary infiltration, histological proof of vasculitis and mono- or poly-neuropathy. We report here on a 45-year-old male who developed erythematous macules, papules and hemorrhagic vesicles on both right extremities along with a tingling sensation and sacral pain. He has been suffering from recurrent allergic rhinitis and bronchial asthma for 6 months. The laboratory findings showed severe eosinophilia (22.3\%), hyper-IgE and positivity for p-ANCA. On the histological examination of the hemorrhagic vesicle on the right lower leg, leukocytoclsatic vasulitis and many neutrophils and eosinophils around the cutaneous vessels were observed in the dermis. (Ann Dermatol 21(2) 213 216, 2009)

\section{-Keyword-}

Churg-Strauss syndrome (CSS)

\section{INTRODUCTION}

Churg-Strauss syndrome (CSS) or allergic granulomatosis angiitis is a rare primary vasculitic disease that's characterized by late-onset asthma, multisystemic involvement and an eosinophilic vasculitis that affects the small and medium sized vessels ${ }^{1}$. This syndrome was first described in 1951 by Churg and Strauss ${ }^{2}$. Most CSS patients are

Received August 12, 2008, Accepted for publication December 15, 2008

Reprint request to: Cheol Heon Lee, M.D., Department of Dermatology, Kangnam Sacred Heart Hospital, College of Medicine, Hallym University, 948-1, Daelim1-dong, Youngdeungpo-gu, Seoul 150-950, Korea. Tel: 82-2-829-5221, Fax: 82-2-832-3237, Email: dermlee@ yahoo.com adults in the third and fourth decades of life and there is a slight male predominance ${ }^{2}$. Although a number of classification systems have been proposed, the American College of Rheumatology (ACR) classification has been accepted as a representative model ${ }^{3,4}$. The ACR proposed the following 6 classification criteria, and 4 of which must be present for making the diagnosis of CSS: asthma, eosinophilia greater than $10 \%$, paranasal sinusitis, pulmonary infiltration, histological proof of vasculitis and mono- or poly-neuropathy. Using these criteria result in $85 \%$ sensitivity and $99.7 \%$ specificity to diagnose CSS. Herein, we report on a case of CSS that was confirmed by the ACR classification.

\section{CASE REPORT}

A 45-year old male was admitted for having developed tingling sensations in his right hand and right lower extremity along with sacral pain over the course of 1 month. The patient presented with malaise, night fever, cough, sputum, anorexia, dyspepsia and a recent weight loss of 6 $\mathrm{kg}$ within the 2 months immediately prior to admission. He had been suffering from allergic rhinitis for several years, chronic active gastritis for 2 years and bronchial asthma for 6 months.

The physical examination revealed a moderate fever of $37.9^{\circ} \mathrm{C}$ but the vital signs were stable and there were motor and sensory deficits involving the right distal upper and lower extremities. The skin examination revealed erythematous macules, papules and hemorrhagic vesicles on both right extremities (Fig. 1).

The laboratory findings showed an elevated white blood cell (WBC) count of $13.2 \times 10^{9} / \mathrm{L}$ (reference range: $4 \sim$ $10 \times 10^{9} / \mathrm{L}$ ) with a differential count of neutrophils $63.5 \%$, eosinophils $22.3 \%$, lymphocytes $12.0 \%$, monocytes $1.4 \%$ and basophils $0.3 \%$, an elevated absolute eosinophil count of $2.94 \times 10^{9} / \mathrm{L}$ (reference range: $0 \sim 0.5 \times 10^{9} / \mathrm{L}$ ), an elevated erythrocyte sedimentation rate (ESR) of $75 \mathrm{~mm} / \mathrm{h}$ (reference range: $1 \sim 15 \mathrm{~mm} / \mathrm{h}$ ), an elevated C-reactive 
protein (CRP) level of $158.8 \mathrm{mg} / \mathrm{dl}$ (reference range: $0 \sim 3$ $\mathrm{mg} / \mathrm{dL}$ ), an elevated rheumatoid factor level of $86.4 \mathrm{IU} / \mathrm{ml}$

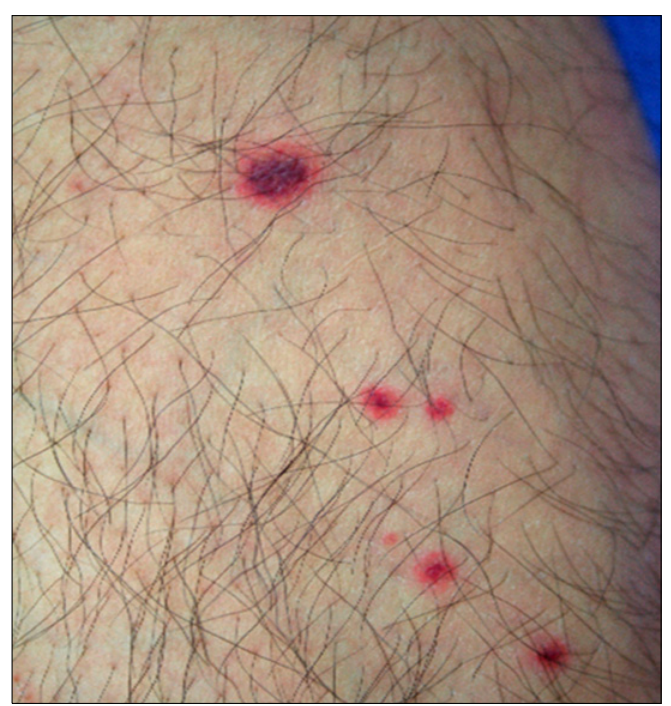

Fig. 1. Erythematous macules with central hemorrhagic vesicles on the right lower leg. (reference range: $0 \sim 20 \mathrm{IU} / \mathrm{ml}$ ), and an elevated IgE level of $2726.4 \mathrm{IU} / \mathrm{ml}$ (reference range: $0 \sim 378 \mathrm{IU} / \mathrm{ml}$ ). The prothrombin time (PT) was slightly prolonged to 13.1 seconds (reference range: $10.3 \sim 12.5$ seconds), the activated partial thromboplastin time (aPTT) was in the normal range of 28.7 seconds, the platelet count was in the normal range of $133 \times 10^{3} / \mathrm{UL}$ and the antithrombin III was mildly decreased to $78 \%$ (reference range: $80 \sim 120 \%$ ). The patient was positive for P-ANCA, but he was negative for C-ANCA and antinuclear antibody. The C3, IgA, IgG and IgM levels were within the normal ranges, but the $\mathrm{C} 4$ level was increased to $79.5 \mathrm{mg} / \mathrm{dl}$ (normal range: $10 \sim 40 \mathrm{mg} / \mathrm{dl}$ ). The serology studies for hepatitis $B$ and toxoplasmosis as well as for antibodies to HIV were negative and the VDRL was non-reactive.

The plain X-rays of the chest and paranasal sinuses showed no active infiltration, but a chest CT showed old inflammatory lesions in the left lower lung, the lingular segment and the right middle lobe. Protein electrophoresis showed a normal value for the serum protein and albumin, but there were slightly increased urine protein and
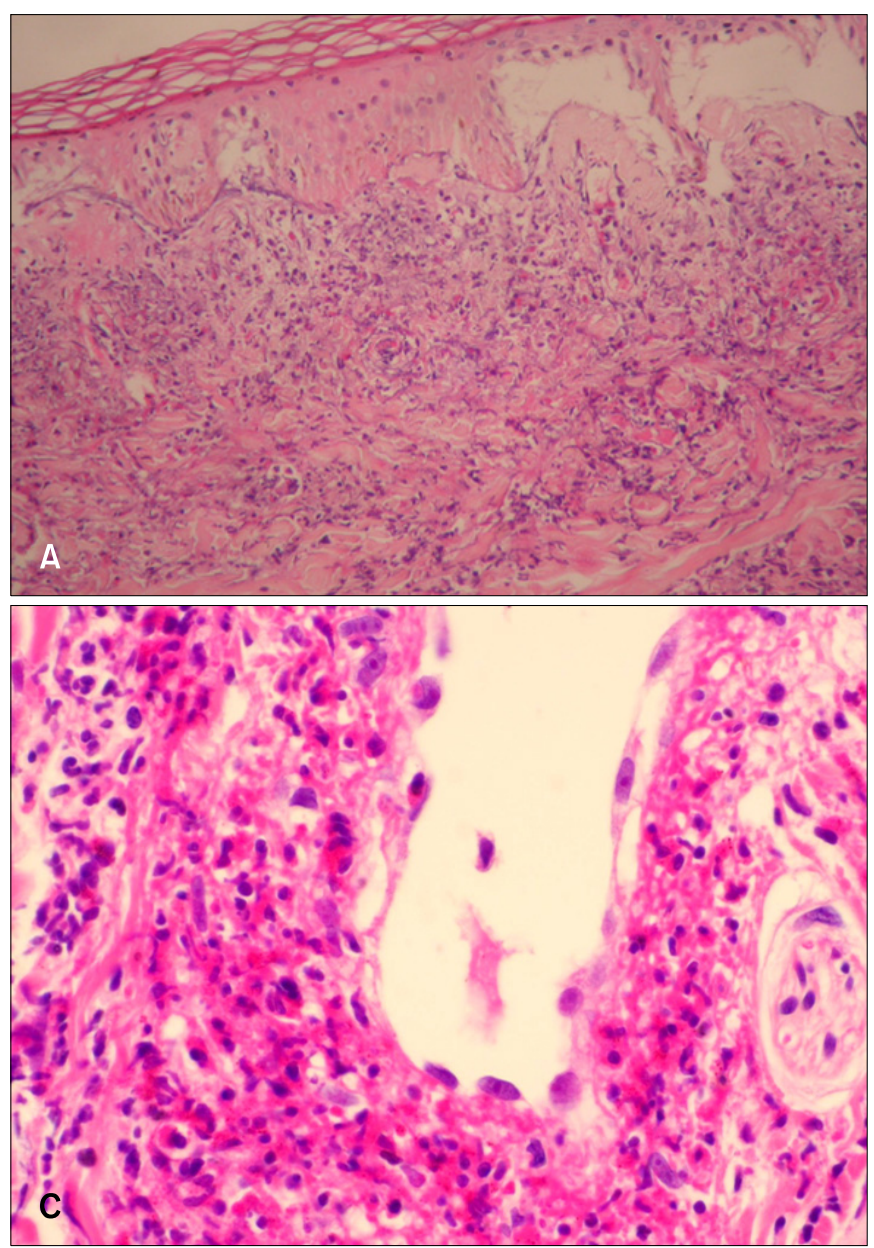

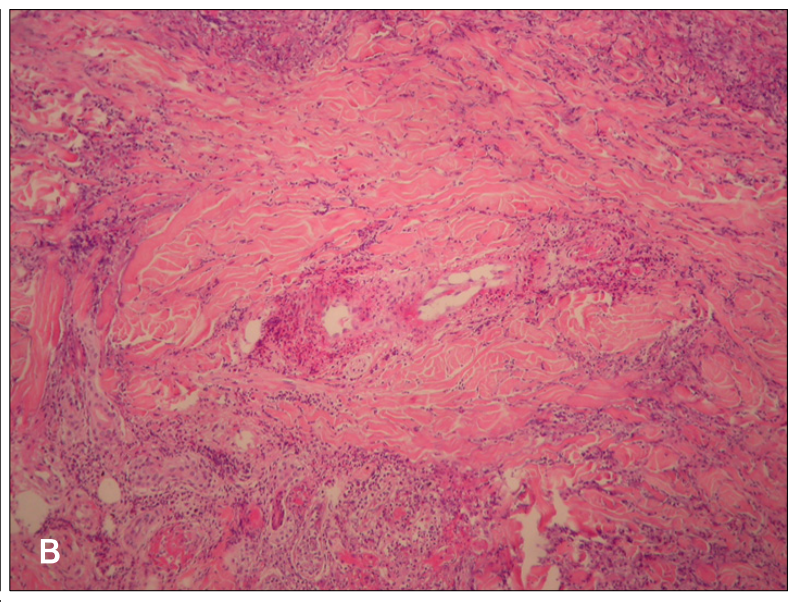

Fig. 2. (A) Biopsy of a hemorrhagic vesicle on the right lower leg shows sub-epidermal bulla and a perivascular leukocytoclstic infiltration with much nuclear dust in the upper dermis (H\&E stain, $\times 200)$. (B) Many inflammatory cells are infiltrated in the middle \& lower dermis, and especially around the perivascular area (H\&E stain, $\times 100$ ). (C) The perivascular infiltration consists of many neutrophils, lymphocytes, histiocytes, neutrophil fragments and eosinophils (H\&E stain, $\times 400)$. 
albuminuria. IVP was performed and it showed that the kidney, bladder and urinary tracts were normal. Electromyography was also performed, and demyelinating polyneuropathy was suggested by the results.

We performed hematoxyline-eosin staining on a section of a skin biopsy specimen from a hemorrhagic vesicle on the right lower leg. There were sub-epidermal bulla and perivascular leukocytoclstic infiltrations with much nuclear dust in the upper dermis. Additionally, there were diffuse inflammatory cell infiltrations in the middle \& lower dermis, and especially in the perivascular area. Many neutrophils, lymphocytes, neutrophil fragments and eosinophils were observed around the cutaneous vessels (Fig. 2). The Giemsa staining and Gram staining were both negative.

The patient was diagnosed with CSS and he was treated with a high dose of oral corticosteroid (predinisolone 1 $\mathrm{mg} / \mathrm{kg}$ ) for 18 days with improvement of his symptoms and laboratory findings. Before the treatment with oral corticosteroid, the WBC count was $13.29 \times 10^{9} / \mathrm{L}$ with the differential count of eosinophils $42.3 \%$, the absolute eosinophil count was $5.62 \times 10^{9} / \mathrm{L}$, the ESR was $97 \mathrm{~mm} / \mathrm{h}$, the CRP level was $120.6 \mathrm{mg} / \mathrm{dl}$ and the PT was 12.9 seconds. After 3 days of treatment, the WBC count decreased to $11.96 \times 10^{9} / \mathrm{L}$ with a differential count of eosinophils $0.9 \%$, the absolute eosinophil count dropped to $0.11 \times 10^{9} / \mathrm{L}$, the PT decreased to 11.7 seconds; after 5 days of treatments, the CRP level decreased to 12.5 $\mathrm{mg} / \mathrm{dL}$. After 2 weeks of treatments, the patient's skin lesions and tingling sensations were much improved, but his sacral pain was only slightly improved.

\section{DISCUSSION}

Churg-Strauss syndrome (CSS) is a multisystem disease that's characterized by asthma, peripheral eosinophilia, peripheral neuropathy, pulmonary infiltrates and paranasal sinus abnormalities. CSS can affect virtually any organ system in the body, although occasionally one organ system is predominantly affected ${ }^{5}$. Skin lesions are an important feature of CSS because they are one of the most common extra-pulmonary findings, and these occur in up to $50 \sim 70 \%$ of the cases ${ }^{1,3,6}$. The main types of skin lesions found in CSS include (1) erythematous maculopapules resembling erythema multiforme; (2) hemorrhagic lesions ranging from petechiae to extensive ecchymosis, and these lesions are often associated with wheals, necrosis and ulceration, and (3) cutaneous and subcutaneous nodules that are usually deep-seated and tender with a predilection for the scalp and temple region ${ }^{7}$. Other skin findings include livedo reticularis, papulovesicles, non-pit- ting periorbital edema and nail-fold infarcts ${ }^{8,9}$. The clinical appearance of the skin lesions may be variable; however, their identification, together with some of the other typical clinical features of the disease, will lead to an early diagnosis of CSS. Other manifestations include pulmonary infiltrates, peripheral neuropathy (e.g., mononeuritis multiplex), cardiac involvement (e.g., eosinophilic endomyocarditis or coronary vasculitis) and renal involvement (e.g., glomerulonephritis). Among the 7 cases reported in the Korean Journal of Dermatology, most of them had a medical history of chronic rhinitis or asthma, and the patients showed general symptoms, including malaise and fever with peripheral neuropathy ${ }^{10,11}$. Our patient also had a medical history of chronic rhinitis and asthma and he showed general symptoms of malaise and fever. Most of the previously reported cases had peripheral neuropathy that was localized to the digital extremities, but this case showed peripheral neuropathy not only in both right digital extremities, but also in the sacral area.

CSS is compatible with histological palisading granulomas in association with vessel-based changes. For most cases of CSS, the hemorrhagic lesions show leukocytoclastic vasculitis (LCV) and the dermis shows a granuloma reaction called palisading necrotizing granuloma, which is predominantly composed of arranged histiocytes and multinucleated giants cells that are centered around degenerated collagen fibers. These palisading granulomas can be surrounded by diffuse inflammatory exudates that are rich in eosinophils. However, these granulomas are not always present, so they must not become a requirement for the diagnosis of $\mathrm{CSS}^{12}$. Despite this, there was a previously reported case in which palisading granulomas were not detected in multiple skin biopsies ${ }^{13}$. In our present case, specific palisading granulomas were not evident.

CSS is considered to be a TH2-mediated disease ${ }^{14}$. Activated $\mathrm{T}_{\mathrm{H}} 2$ lymphocytes play a central role through their production of cytokines such as interleukin 4, 5 and 13. These cytokines mediate the accumulation of mast cells and basophils and especially eosinophils. One study suggested that the IgE levels as well as the eosinophil counts have a pathological central role in $\mathrm{CSS}^{7}$. Therefore, assessing the eosinophil count and measuring the IgE level may be useful to follow the disease activity and the response to therapy. ANCA-positivity is correlated with more vasculitic features, and some of the reported data suggests that the ANCA levels are correlated with the CSS disease activity ${ }^{14-16}$. Central nervous system involvement, which is one of the most severe disease manifestations associated with a poor prognosis, has been more commonly reported in P-ANCA positive patients ${ }^{15}$.

Before the introduction of immunosuppressive systemic 
treatment, the length of survival after the diagnosis of CSS was only a few months. Today, oral corticosteroids and immunosuppressants (cyclophosphamide and azathioprine) have significantly increased the survival rate and the length of survival. However, there are significant side effects with administering steroid and/or immunosuppressants that frequently appeared with their continuous or repeated use. Thus, there are some alternative immunosuppressive drugs such as interferon- $\alpha$, mycophenolate mofetil and leukotriene receptor antagonist (e.g., montelukast, pranlukast, and zafirlukast) that have been shown to be both efficacious and safe ${ }^{7,17-19}$. Our current case also showed a good response to oral corticosteroids, but other immunosuppressant drugs are probably necessary to treat the patient's sacral pain.

\section{REFERENCES}

1. Lanham JG, Elkon KB, Pusey CD, Hughes GR. Systemic vasculitis with asthma and eosinophilia: a clinical approach to the Churg-Strauss syndrome. Medicine (Baltimore) 1984;63: 65-81.

2. Churg J, Strauss L. Allergic granulomatosis, allergic angiitis, and periarteritis nodosa. Am J Pathol 1951;27:277-301.

3. Masi AT, Hunder GG, Lie JT, Michel BA, Bloch DA, Arend WP, et al. The American College of Rheumatology 1990 criteria for the classification of Churg-Strauss syndrome (allergic granulomatosis and angiitis). Arthritis Rheum 1990;33:10941100.

4. Jennette JC, Falk RJ, Andrassy K, Bacon PA, Churg J, Gross $W L$, et al. Nomenclature of systemic vasculitides. Proposal of an international consensus conference. Arthritis Rheum 1994;37:187-192.

5. Gayraud M, Guillevin L, le Toumelin P, Cohen P, Lhote F, Casassus $\mathrm{P}$, et al. Long-term followup of polyarteritis nodosa, microscopic polyangiitis, and Churg-Strauss syndrome: analysis of four prospective trials including 278 patients. Arthritis Rheum 2001;44:666-675.

6. Guillevin L, Cohen P, Gayraud M, Lhote F, Jarrousse B, Casassus P. Churg-Strauss syndrome. Clinical study and long-term follow-up of 96 patients. Medicine (Baltimore)
1999;78:26-37.

7. Kawakami T, Soma Y, Kawasaki K, Kawase A, Mizoguchi M. Initial cutaneous manifestations consistent with mononeuropathy multiplex in Churg-Strauss syndrome. Arch Dermatol 2005;141:873-878.

8. Davis MD, Daoud MS, McEvoy MT, Su WP. Cutaneous manifestations of Churg-Strauss syndrome: a clinicopathologic correlation. J Am Acad Dermatol 1997;37:199-203.

9. Schwartz RA, Churg J. Churg-Strauss syndrome. Br J Dermatol 1992;127:199-204.

10. Im M, Nam YH, Seo YJ, Lee JH, Park JK. A case of Churg-Strauss syndrome showing severe skin necrosis. Korean J Dermatol 2006;44:216-219.

11. Lee SW, Park JS, Woo MJ, Kim SW. A case of Churg-Strauss syndrome showing hemorragic bullae. Korean J Dermatol 2003;41:1370-1373.

12. Elder DE, Elenitsas R, Johnson BL Jr, Murphy GF, editors. Lever's histopathology of the skin. 9th ed. Philadelphia: Lippincott Williams \& Wilkins, 2005:223.

13. Chang SE, Cheong JK, Choi JH, Sung KJ, Moon KC, Koh JK. An unusual case of Churg-Strauss syndrome. Ann Dermatol 1998;10:167-171.

14. Termeer CC, Simon JC, Schopf E. Low-dose interferon al$\mathrm{fa}-2 \mathrm{~b}$ for the treatment of Churg-Strauss syndrome with prominent skin involvement. Arch Dermatol 2001;137: 136-138.

15. Guillevin L, Visser H, Noel LH, Pourrat J, Vernier I, Gayraud $\mathrm{M}$, et al. Antineutrophil cytoplasm antibodies in systemic polyarteritis nodosa with and without hepatitis B virus infection and Churg-Strauss syndrome--62 patients. J Rheumatol 1993;20:1345-1349.

16. Solans R, Bosch JA, Perez-Bocanegra C, Selva A, Huguet $P$, Alijotas J, et al. Churg-Strauss syndrome: outcome and long-term follow-up of 32 patients. Rheumatology (Oxford) 2001;40:763-771.

17. Tervaert JW, Kallenberg CG. Anti-myeloperoxidase antibodies in Churg-Strauss syndrome. J Neurol 1993;240:449450.

18. Assaf C, Mewis G, Orfanos CE, Geilen CC. Churg-Strauss syndrome: successful treatment with mycophenolate mofetil. Br J Dermatol 2004;150:598-600.

19. Tang MB, Yosipovitch G. Acute Churg-Strauss syndrome in an asthmatic patient receiving montelukast therapy. Arch Dermatol 2003;139:715-718. 